\title{
UNA MIRADA SOBRE LAS POLÍTICAS PÚBLICAS DEL MERCOSUR EN MATERIA DE CULTURA*
}

Ma. Alfonsina Baumann*

\section{Resumen}

La conformación inicial del Mercosur que proponía abordar la integración económica implicó, indefectiblemente, el avance en el plano de la integración política.

En la década del noventa, bajo el lema de mayor competitividad y crecimiento orientado a alcanzar un "efecto derrame", operó el relajamiento de controles públicos y flexibilidad normativa. Esto conllevó notables beneficios empresariales, pero sin impacto positivo en el conjunto de la población. Paulatinamente fueron surgiendo nuevos enfoques dirigidos a tomar otros rumbos y alentar un "nuevo intervencionismo" para socializar las pérdidas generadas por la crisis. Desde esa perspectiva se volvió necesario orientar en lo político, sobre bases constitucionales, una reconversión y abordar nuevos ejes que posibilitaran avanzar en integración.

Progresivamente en el bloque, la dimensión social -la cual se relaciona con la dimensión cultural- fue incorporando "agendas de integración" sobre medio ambiente, cohesión social, participación ciudadana, movimiento de personas, entre otras.

Este artículo describe ciertas notas características que evidencian el proceso de integración en el plano cultural del Mercosur y destaca perspectivas teóricas del campo del Derecho y las Relaciones Internacionales desde las que se pueden pensar herramientas que incorporen procesos participativos, en pos de garantizar nuevos derechos y asegurarlos en condiciones de igualdad entre generaciones presentes y futuras.

Palabras clave: Integración - Cultura - Derechos Culturales - Derechos Humanos Políticas - Tutela - Gobernanza

\section{A LOOK AT MERCOSUR PUBLIC POLICIES IN THE FIELD OF CULTURE}

\begin{abstract}
The initial formation of Mercosur that proposed to tackle economic integration inevitably implied progress in the area of political integration.

In the nineties, under the motto of greater competitiveness and growth aimed at

\footnotetext{
* Investigación realizada en el marco de la tesis de la autora en la Maestría en Integración y Cooperación Internacional de la UNR-CEI-CERIR, titulada: "Los avances del Mercosur en la dimensión cultural: Su impacto en la tutela del patrimonio cultural arquitectónico en la República Argentina en el período 2014-2018”.

** Abogada. Facultad de Ciencias Jurídicas y Sociales, Universidad Nacional del Litoral. Magíster en Integración y Cooperación Internacional CEI-CERIR, UNR. Correo electrónico: estudiobaumann@aol.com
} 
achieving a "spillover effect", the relaxation of public controls and regulatory flexibility took place. This brought significant business benefits but no positive impact on the population as a whole. Gradually new approaches were emerging aimed at taking other directions and encouraging a "new intervention" to socialize the losses generated by the crisis. From that perspective, it became necessary to guide politically, on constitutional grounds, a reconversion and address new axes that enabled progress in integration.

In the area of social dimension -which relates to the cultural dimension"Integration agendas" on the environment, social cohesion, citizen participation, movement of people, among others, were incorporated.

This article describes certain characteristic notes that evidence the process of integration in the cultural sphere and highlights theoretical perspectives in the field of Law and International Relations from which can be thought tools that incorporate participatory processes, in order to guarantee new rights and secure them in conditions of equality between present and future generations.

Key words: International Integration - Culture - Cultural Rights - Human Rights Policies - Legal Protection - Governance

TRABAJO RECIBIDO: 11/03/2020 - TRABAJO ACEPTADO: 02/05/2020 


\section{Introducción}

Durante el siglo XX, a lo largo del mundo y como uno de los elementos constitutivos de las sociedades modernas, la cultura inició un paulatino proceso de legitimación en el discurso público y en la agenda política de los Estados.

En el ámbito de las Relaciones Internacionales se lograron diversas expresiones en declaraciones y acuerdos en organismos internacionales, las cuales han impactado en la realidad particular de cada país y en sus espacios de participación.

Un hito de importancia fundamental lo constituye la incorporación de la cultura como un derecho en la "Declaración Universal de los Derechos Humanos", aprobada por la Organización de Naciones Unidas en 1948. En la misma, la defensa del ejercicio y la libertad en materias culturales se expresa fundamentalmente en su artículo 22, el cual enuncia que las personas, como miembros de la sociedad, tienen el derecho a " (...) la satisfacción de los derechos económicos, sociales y culturales, indispensables a su dignidad y al libre desarrollo de su personalidad". En el mismo sentido, la "Declaración de la Unesco sobre Diversidad Cultural", adoptada en el año 2001, en su artículo 5 encuadra a los Derechos Culturales como parte integrante de los Derechos Humanos, y en tal sentido, los declara “(...) universales, indisociables e interdependientes".

La Observación $\mathrm{N}^{\circ} 21$ del año 2009 del Comité de Derechos Económicos, Sociales y Culturales señala que la cultura "(...) comprende, entre otras cosas, las formas de vida, el lenguaje, la literatura escrita y oral, la música y las canciones, la comunicación no verbal, los sistemas de religión y de creencias, los ritos y las ceremonias, los deportes y los juegos, los métodos de producción o la tecnología, el entorno natural y el producido por el ser humano, la comida, el vestido y la vivienda, así como las artes, las costumbres y tradiciones, por los cuales los individuos, grupos y comunidades expresan su humanidad, y el sentido que dan a su existencia, y configuran una visión del mundo que representa su encuentro con las fuerzas externas que afectan a sus vidas".

El Mercosur, como espacio de integración, no fue ni es ajeno a estos reconocimientos y reivindicaciones, desde el momento en el que son los Estados que lo conforman quienes llevan adelante las acciones necesarias para garantizar los derechos establecidos en estos instrumentos jurídicos.

\section{1- La dimensión cultural en el Mercosur.}

Los Estados Parte del Mercosur han suscripto y se han obligado, en mayor o menor medida, a los postulados y disposiciones de diversos instrumentos jurídicos internacionales en lo concerniente a la tutela de Derechos Culturales.

El bloque, hace más de dos décadas, inició paulatinamente un camino en torno al reconocimiento de la cultura como elemento constitutivo del proceso de integración. Este camino comenzó, primeramente, a partir de la concreción de diversas reuniones especializadas de Ministros o Responsables del Área Cultural, la primera de las cuales se llevó a cabo en Buenos Aires, en marzo de 1995 y, la segunda, en Asunción en julio 
del mismo año. A partir de allí se crearon cuatro Comisiones Técnicas: Patrimonio, Industrias Culturales, Capacitación y Redes de Información, junto a un Comité Coordinador General. Finalmente, se adicionó una reunión de Ministros de Cultura, como foro negociador de alto nivel.

La Decisión N¹1/96 del Consejo del Mercado Común suscripta por Argentina, Brasil, Uruguay y Paraguay creó el Protocolo de Integración Cultural del Mercosur, aprobado en diciembre de 1996. En esta norma se fijaron los principios y orientaciones del denominado "Mercosur Cultural", como ámbito específico para llevar adelante las políticas en materia de cultura y, de esta manera, asegurar su institucionalidad. En el año 2008, la República Argentina, la República Federativa de Brasil, la República de Paraguay, la República de Uruguay, la República de Bolivia, la República de Ecuador, la República de Colombia, la República del Perú y la República Bolivariana de Venezuela, reafirmaron la importancia de los instrumentos y acciones llevadas a cabo en materia de cultura en el ámbito de la UNESCO, reivindicando a la cultura como elemento esencial en los procesos de integración. En este sentido, proclamaron la "Declaración de Integración Cultural del Mercosur" para apuntalar las acciones políticas en ese aspecto.

\section{2- La puesta en valor de las políticas culturales en el Mercosur}

El Mercosur como sujeto colectivo de tinte multicultural necesita, entre otras medidas, de una política cultural, es decir, un conjunto de principios teóricos y operativos que orienten sus acciones (VIVA, 2011:164).

Entendemos que no obstante los vaivenes económicos, la cultura apuntala la dimensión política de la integración, ya que contribuye a construir una conciencia de ciudadanía Mercosur. Esta concepción no viene a reemplazar la de las ciudadanías nacionales, sino que las complementa y las amplía al revalorizar el papel de las instituciones nacionales y supranacionales como presencia conjunta de la región en el escenario internacional.

Por otra parte, en la dimensión cultural se vislumbran nuevos enfoques sobre la acción pública en poblaciones deterioradas y que enriquecen las miradas sobre la pobreza y las prácticas sociales, como consecuencia de la acumulación de evidencia empírica de las ciencias sociales en estas últimas décadas. Se complejiza, así, la visión sobre la pobreza y la exclusión social revelando como como muy productivo el trabajo cultural para regenerar sentidos colectivos (WORTMAN, 2015).

En este orden de ideas, el desarrollo de una política cultural coherente y con continuidad en el Mercosur podría convertirse en una estrategia que, en última instancia, podría redundar en cohesión social, desarrollo económico, expansión comercial e influencia política internacional. Aparecen así los ciudadanos como parte necesaria en la formulación de las políticas culturales, y como protagonistas en la construcción de realidades que impactan positivamente si se dirigen a reducir las asimetrías.

Si pensamos en América del Sur como una región inestable, la asunción de compromisos "serios" entre socios en el marco de la integración, podría significar un reaseguro para el sistema democrático, en tanto lleva a estimular el desarrollo de mecanismos de protección y seguridad colectiva para la democracia de sus miembros (GRINSPUN, 2009). 
En este plano encontramos cierto grado de avances en políticas culturales vinculadas a las tecnologías de la información y la comunicación (TIC). Entre estas se destacan:

$\checkmark$ En el marco de la difusión del Mercosur Cultural, se instauró el Sistema de Información Cultural del Mercosur (SICSUR) en el año 2006, con el fin de disponer de una carga de datos sobre cultura sudamericana.

$\checkmark$ El "Programa Puntos de Cultura" implementado por países del Mercosur Uruguay, Paraguay, Brasil, Perú, Chile, Ecuador y Argentina- del año 2009 (MIHAL, 2014)

$\checkmark$ El "Programa Mercosur Audiovisual", resultado de un convenio de cooperación de la Unión Europea y el Mercosur y desarrollado en el ámbito de la Reunión Especializada de Autoridades Cinematográficas y Audiovisuales del Mercosur (RECAM), del año 2014

$\checkmark$ En el campo de la cooperación cultural en el Mercosur, en 2017, se suscribió un Memorando de Entendimiento entre el Ministerio de Cultura de Argentina y el Ministerio de Cultura de Perú -con una duración de 5 años-, con el objeto de facilitar el intercambio de artistas en artes audiovisuales y de multimedia, artes digitales y posdigitales, artes plásticas, gestión cultural, sector editorial, entre otros.

No obstante los avances destacados en materia de cultura, los mismos no se constituyen como políticas consistentes y uniformes, debido a que no se observan acciones que aborden todos los ejes temáticos del plano cultural.

\section{3- Un enfoque de cultura con perspectiva de Derecho Humano}

Como señaláramos en la introducción, en el plano jurídico, la cultura tiene fuerte raigambre en la normativa de Derechos Humanos.

América Latina, históricamente reconoció la diversidad y los Derechos Culturales. Primigeniamente, lo hizo a través de la "Declaración Universal de los Derechos Humanos" de 1948 (MEJÍA, 2004). Consecutivamente, los países de la región firmaron el "Pacto Internacional de Derechos Económicos Sociales y Culturales" del año 1966, el cual amplió y especificó la tutela de estos derechos responsabilizando especialmente a los Estados en materia de garantía de los mismos. En el ámbito continental la "Declaración Americana de los Derechos y Deberes del Hombre" de 1948 se constituyó en piedra angular del Sistema Interamericano de Protección de los Derechos Humanos. Este instrumento introdujo los Derechos Culturales en su artículo 13 bajo el título "Derecho a los beneficios de la cultura". Finalmente, los Estados latinoamericanos firmaron la "Convención Americana sobre Derechos Humanos" de 1969 (Pacto de San José de Costa Rica) y el "Protocolo adicional a la Convención Americana sobre Derechos Humanos en materia de Derechos Económicos, Sociales y Culturales" (Protocolo de San Salvador de 1988), consagrando de esta manera los Derechos Culturales como Derechos Humanos fundamentales (ALONSO y FIDEL, 2017). En este orden, el 4 de junio de 2012 la Organización de los Estados Americanos (OEA) aprobó una resolución que obliga a los Estados parte del Protocolo de San Salvador a presentar informes nacionales de avance hacia la realización de los Derechos Económicos, Sociales y Culturales mediante el uso de indicadores en materia de 
seguridad social, $\quad$ salud $\quad y \quad$ educación (http://www.oas.org/es/sedi/ddse/documentos/Protocolo\%20San\%20Salvador/AG2012. pdf).

En concordancia con ello, creemos indispensable reseñar las políticas implementadas desde el Mercosur para la tutela de la cultura desde la perspectiva de los Derechos Humanos, es decir, establecer puntos de conexión con el Mercosur Cultural en cuanto espacio específico para el abordaje.

En agosto del año 2006, en el marco de la V Reunión de Altas Autoridades sobre Derechos Humanos y Cancillerías del MERCOSUR y Estados Asociados (RAADH), se aprobó la creación del Grupo de Trabajo sobre Educación y Cultura en Derechos Humanos. En el año 2009, la RAADH decide elevar la misma a Comisión Permanente.

Si bien existe labor destacada del bloque en materia de Derechos Humanos, en el trabajo de la Comisión el tema educativo suele ser prioritario frente a otros. En este sentido y en base a las líneas de intervención y documentos disponibles de la Comisión Permanente Educación y Cultura en Derechos Humanos, no pudimos constatar el tratamiento de los Derechos Culturales como materia específica (RAADH - COMISIÓN PERMANENTE EDUCACIÓN Y CULTURA EN DERECHOS HUMANOS).

En el plano de la difusión, el Mercosur inició en el año 2015 un plan de comunicación institucional llamado "Plan de Comunicación y Cultura" del Instituto de Políticas Públicas en Derechos Humanos (IPPDH), con el propósito de difundir las acciones en la temática de Derechos Humanos y sugerir, entre otras cuestiones, la intervención de actores, la ampliación del público en las redes sociales y la articulación interinstitucional, con el objetivo de contribuir a la agenda de comunicación en Derechos Humanos, incluyendo acciones culturales y apoyo comunicacional a la RAADH.

Asimismo, en el aspecto normativo, el Mercosur lanzó -con apoyo de la Organización de Estados Iberoamericanos (OEI)- unas Directrices Regionales de Educación y Cultura en Derechos Humanos. Las mismas están dirigidas a generar políticas nacionales en la materia y a definir acciones regionales que deben ser implementadas por las instituciones del Mercosur, identificando criterios compartidos de los Estados Parte y coordinando las políticas públicas. A su vez, en este ámbito se recibieron aportes de organizaciones sociales a través de la IV Consulta del Foro de Participación Social del IPPDH y, en el mes de junio de 2017, la Comisión Permanente de Educación y Cultura en Derechos Humanos de los Estados Parte y Asociados aprobó un documento que resumió estos aportes (IPPDH - Informe anual 2017).

Por otro lado, del Informe de Gestión del IPPDH del Primer Semestre de 2018, si bien trabaja sobre el mandato de la Comisión para determinados colectivos sociales en la agenda cultural, no hay mención sobre la participación activa de grupos enfocados en temas ambientales o de defensa del patrimonio cultural (IPPDH - Informe Primer Semestre 2018).

En relación a los datos obtenidos de las Altas Reuniones de sobre Derechos Humanos del Mercosur llevadas a cabo durante el período 2014-2018 observamos que, desde la perspectiva de los Derechos Humanos, se vislumbró cierto grado de interés, al menos teórico, en temas de cultura. Como se dijera precedentemente, estas refirieron principalmente aspectos de difusión y comunicación interna (RAADH). 


\section{4- Aportes de las Relaciones Internacionales para el desarrollo de las políticas culturales del Mercosur}

El Mercosur como ámbito de acentuado corte presidencialista hace posible pensar el rediseño de un sistema de gobernanza capaz de incorporar elementos brindados desde las diferentes perspectivas teóricas del campo de las Relaciones Internacionales que permitan redefinir estrategias de acción para el desarrollo de las políticas culturales.

Las teorías Posmodernistas, críticas de las posiciones positivistas parten de establecer relaciones entre conocimiento, historia, cultura y relaciones de poder que permiten avanzar en el proceso de integración cultural. El poder interactuar con otras disciplinas, entendemos, puede proporcionar conceptualizaciones útiles para el análisis (SALOMÓN GONZÁLEZ, 2002)

Teniendo en cuenta que la historia, el lenguaje y las identidades son elementos claves en la integración latinoamericana y que aquellas son procesos de construcción que se van conformando con miradas propias y externas consideramos valiosos los aportes desarrollados en el plano de las Relaciones Internacionales, los cuales conjugados con las diversas perspectivas jurídicas permitirían avanzar en aspectos de protección de la cultura.

Así el "Constructivismo", como perspectiva teórica desde la cual la realidad depende de las ideas y el lenguaje a través de las construcciones o representaciones que llegan de un contexto histórico y social, posibilita reflexionar acerca de "construir", con participación significativa de la sociedad, la orientación y los principios de los procesos de integración en América Latina y dotar de valor a los bienes objeto de tutela y promoción.

En la búsqueda de revalorizar el componente cultural en la agenda de los Estados el Derecho Constitucional podría facilitar, en el campo de las Relaciones Internacionales, el desarrollo de un sistema de "Gobernanza Multinivel" y fundar la adopción de políticas de descentralización y la autogestión en unidades políticas menores (DALLA VÍA, 2004). En este esquema, diversos actores participan simultáneamente en diversos planos: supraestatales, estatales y subestatales, sustrayendo competencias a los Estados-nación y con implicaciones políticas en las identidades territoriales (VIEIRA POSADA, 2005). En la gobernanza multinivel adquiere relevancia la cultura política, la distribución del poder, las instituciones y las capacidades de gestión (MORATA, 2014). Esto permitiría generar consenso político y social para legitimar los procesos y garantizar la confianza de los diversos actores.

Así el esquema Mercosur Cultural puede ser pensado como "proceso de creación" capaz de abrirse a la participación social. Una entidad política en el que la autoridad se reparta entre múltiples actores y niveles de gobierno (HOOGHE \& MARKS, 2004). Una "gobernanza multinivel" permite la complementariedad en la esfera pública al tiempo que da lugar a la participación privada, siendo desde esta perspectiva que cobran relevancia las nociones de "cohesión" y "responsabilidades compartidas" (MORATA, 2002).

El "Intergubernamentalismo Liberal" brinda aproximaciones teóricas útiles en torno al valor de los regímenes y las instituciones internacionales para mitigar los 
efectos perjudiciales de la anarquía. El sistema político comunitario se concibe como un régimen internacional (SALOMÓN GONZÁLEZ, 2002) y los actores estatales adquieren un rol preponderante y las instituciones regionales adquieren relevancia. Compartimos la idea de que el Estado puede ser un lugar de experiencia distributiva y cultural (DE SOUSA SANTOS, 2010).

De esta forma el Mercosur como un "sistema político y régimen internacional" puede ser capaz de asignar una agenda cultural para los Estados. Podría pensarse en una integración regional como mecanismo que permita que las decisiones se mantengan en cabeza de los Estados nacionales, allí donde las preferencias son más homogéneas (MALAMUD, 2011)

\section{Conclusión}

En el complejo escenario del siglo XXI consideramos que el esquema del Mercosur aporta su singularidad, tanto por la pluralidad y diversidad de recursos naturales, como por la importancia estratégica en orden a su localización, las capacidades productivas existentes y la vinculación con otros esquemas internacionales.

Como corolario, consideramos auspicioso que desde el ámbito específico del desarrollo de la cultura, específicamente el Mercosur Cultural, se retome la mirada en orden a profundizar la integración cultural desde la articulación con otros ámbitos del esquema, en este caso, el de los Derechos Humanos, a fin de desplegar de manera interconectada nuevas estrategias que potencien y complementen capacidades y que pueden incorporarse mecanismos que faciliten la implementación de acuerdos en espacios de acción colectiva.

Desde nuestra visión la órbita del Mercosur, y de los Derechos Humanos, los Estados Parte ya encuentran fundamento suficiente para actuar sin dilaciones y potenciar sus capacidades en materia de tutela de los Derechos Culturales.

\section{Bibliografía}

AGUIRRE ARIAS, B. (2007), Del concepto de bien histórico-artístico al de patrimonio cultural. Revista Electrónica DU\&P. Diseño Urbano y Paisaje, Volumen IV $\mathrm{N}^{\circ}$ 11. Centro de Estudios Arquitectónicos, Urbanísticos y del Paisaje Universidad Central de Chile. Disponible en: http://dup.ucentral.cl/pdf/11_bien_historico_artistico.pdf Consulta: 2 de Diciembre de 2019

ALONSO, M. V., y FIDEL, D. (2017), Acerca de los derechos culturales. Revista Latinoamericana de Investigación Crítica. CLACSO, Año 4 (6), p.129-150. Disponible en: http://biblioteca.clacso.edu.ar/clacso/se/20170828111008/ImasC6.pdf Consulta: 2 de Diciembre de 2019

ARBUET-VIGNALI, H. (1996), Mercosur, Balance y Perspectivas. Fundación de Cultura Universitaria, Montevideo. 
ARBUET-VIGNALI, H. (2004), Claves Jurídicas de la Integración en los Sistemas del Mercosur y la Unión Europea. Ed. Rubinzal-Culzoni, Santa Fe.

BASALDUA, R. X. (1996), En Torno al Derecho de la Integración, en EL DERECHO, año XXXIV, Buenos Aires, 6 de junio de págs. 23-25

BASALDUA, R. X. (1999). Mercosur y Derecho de la Integración. Ed. AbeledoPerrot, Buenos Aires.

DALLA VÍA, Alberto R. (2004), El marco jurídico e institucional para la gestión internacional de los actores subnacionales gubernamentales en Argentina. Revista Integración y Comercio INTAL N ${ }^{\circ}$ 21, Julio-Diciembre, Año 8, p. 11-26. Disponible en: https://www.researchgate.net/publication/28095894_El_marco_juridico_e_institucional_ para_la_gestion_internacional_de los_actores_subnacionales_gubernamentales_en_Arge ntina Consulta: 7 de Diciembre de 2019

DECLARACION AMERICANA DE LOS DERECHOS Y DEBERES DEL HOMBRE y CONVENCIÓN AMERICANA SOBRE DERECHOS HUMANOS. Disponible en: http://www.corteidh.or.cr/tablas/3768.pdf Consulta: 5 de Septiembre de 2018

DECLARACIÓN UNIVERSAL DE DERECHOS HUMANOS, 10 de diciembre de 1948. Disponible en: https://www.un.org/es/universal-declaration-human-rights/ Consulta: 3 de Septiembre de 2018

DECLARACIÓN UNIVERSAL SOBRE LA DIVERSIDAD CULTURAL, 2 de noviembre de 2001.

Disponible en: https://unesdoc.unesco.org/ark:/48223/pf0000127162_spa Consulta: 4 de Julio de 2018

DE SOUSA SANTOS, B. (2010), Descolonizar el saber, reinventar el poder, Ed. Trilce, Montevideo

ENCLAVE CULTURAL. (2014) Publicación del SICSUR Ahora es cuando. Sistema de Información Cultural del MERCOSUR Año 4 Nº 3 - Mayo 2014

ENDERE, M. L. (2007), Documento sobre la Legislación Nacional Argentina y su relación con las Convenciones establecidas por UNESCO dentro del Sector Cultura. En Legislaciones en el MERCOSUR relativas a las Convenciones de Cultura aprobadas por la UNESCO. Estudio de la situación actual de Argentina, Brasil, Paraguay y Uruguay. Oficina Regional de Ciencia de la UNESCO para América Latina y el Caribe, Montevideo, p 7-42. Disponible en: https://unesdoc.unesco.org/ark:/48223/pf0000159998 Consulta: 10 de Julio de 2018

GRIMSON, A. (2018), Argentina y sus crisis. Revista Nueva Sociedad, Enero Febrero. Disponible en: https://nuso.org/articulo/argentina-y-sus-crisis/ Consulta: 4 de Septiembre de 2018

GRINSPUN, P. (2009). Temas de política Exterior, comercio y relaciones internacionales. Publicación de la Asociación Profesional del Cuerpo Permanente del Servicio Exterior de la Nación, ISSN-1851-9792, AÑO II, N`2 Septiembre, 25 años de Democracia y Política Exterior Argentina

HOOGHE, L.; MARKS, G. (2004). Gobernanza estatocéntrica y gobernanza multinivel, en. Morata, F (ed.) Gobernanza Multinivel en la Unión Europea, Tirant lo Blanch, Valencia. 
HOREN, B., y Rivarola, M. (2008), Políticas Culturales para el Mercosur. Recuperación y revalorización de los espacios de producción cultural independiente, IX Congreso Argentino de Antropología Social. Facultad de Humanidades y Ciencias Sociales - Universidad Nacional de Misiones. Disponible en: https://www.aacademica.org/000-080/443. Consulta: 5 de Marzo de 2018

LAPALMA, M.L. y LEVRAND, N.E. (2013), ¿Cómo regular lo que percibimos? Avances y dificultades en la tutela del paisaje como bien jurídico. Revista de Derecho Ambiental, Universidad de Palermo, Año II, $\mathrm{N}^{\circ}$ 1, Mayo, p. 73-112. Disponible en www.palermo.edu/derecho/pdf/publicaciones/DA_N2_02.pdf Consulta: 2 de Junio de 2018

LEVRAND, N. (2009). Política Legislativa vs. Diversidad Cultural: el desafío de proteger nuestro Patrimonio Cultural. En G. Sozzo, N. Levrand, M. V. Berros, F. Crisalle, L. Spano Tardivo, V. Haidar, y otros, \& G. Sozzo (Ed.), La Protección del Patrimonio Cultural. Estudios sociojurídicos para su construcción. Ediciones UNL. Secretaría de Extensión, Universidad Nacional del Litoral, Santa Fe.

LUNA RAMIREZ, C. (2009), El constructivismo social: ¿Una teoría para el estudio de la política internacional o un esquema para el análisis de la Política Exterior de los estados? Ponencia presentada en las Jornadas del Área de Relaciones Internacionales: una disciplina en constante movimiento. FLACSO, octubre. Disponible en: https://www.academia.edu/3633878/El_contructivosmo_social_carlos_luna_ramirez

Consulta: 20 de Febrero de 2018

MALAMUD, A. (2011), Conceptos, Teorías y Debates sobre la Integración Regional, Norteamérica, vol.6, $\mathrm{N}^{\circ} 2$, México, julio/diciembre. Disponible en línea: http://www.scielo.org.mx/pdf/namerica/v6n2/v6n2a8.pdf Consulta: 23 de Marzo de 2018

MEJÍA, J.L. (2004). ¿Derechos sin Estado? Tres momentos de institucionalidad cultural en América Latina. Revista Pensar Iberoamérica, OEI, $\mathrm{N}^{\circ} 7$, septiembrediciembre. Disponible en: https://www.oei.es/historico/pensariberoamerica/ric07a05.htm Consulta: 8 de Noviembre de 2019

MIHAL, I. (2014), Inclusión digital y gestión cultural en el Mercosur: el Programa Puntos de Cultura. Revista Otras Versiones, Editorial Universidad Autónoma Metropolitana, $\quad \mathrm{N}^{\circ} 34$, septiembre-octubre, p. 126-137. Disponible en: http://sgpwe.izt.uam.mx/files/users/uami/ana/IvanaMihalPuntosCultura.pdf Consulta: 25 de Noviembre de 2019

MONTESINOS PADILLA, C. (2016), Tutela Multinivel de los derechos: concepto, marco teórico y desafíos actuales. Eunomia: Revista Cultural en la Legalidad, $\mathrm{N}^{\circ} 11$, Biblioteca Universidad Carlos III de Madrid, octubre-marzo, p. 211-220. Disponible en: https://e-revistas.uc3m.es/index.php/EUNOM/article/view/3288 Consulta: 23 de Noviembre de 2019

MORATA, F. (2002), "La gobernanza multinivel europea", VII Congreso Internacional del CLAD sobre la Reforma del Estado y de la Administración Pública, Lisboa, Portugal, 8-11 Octubre. Disponible en línea: https://fcpolit.unr.edu.ar/blogs/administracionpublica2/files/2017/04/GobernanzaMultinivel.pdf Consulta: 23 de Marzo de 2018 
PACTO INTERNACIONAL DE DERECHOS ECONÓMICOS SOCIALES Y CULTURALES, 16 de diciembre de 1966. Disponible en: http://servicios.infoleg.gob.ar/infoleginternet/anexos/20000-24999/23782/norma.htm Consulta: 8 de Junio de 2018

PISARELLO, G. (2012), El constitucionalismo social ante la crisis: entre la agonía y la refundación republicano democrática. Revista Derecho del Estado. $\mathrm{N}^{\circ} 28$, junio, p. 55-75. Disponible en: https://revistas.uexternado.edu.co/index.php/derest/article/view/3179 Consulta: 15 de Diciembre de 2019

SANAHUJA, J. A. (2017), Regionalismo e integración en América Latina: de la fractura Atlántico Pacífico a los retos de una globalización en crisis, Revista Pensamiento Propio, Investigación y Análisis $\mathrm{N}^{\circ} 44$, p.29-76. Disponible en: www.cries.org/wpcontent/uploads/2017/02/007-sanahuja.pdf Consulta: 17 de Octubre de 2018

SALOMÓN GONZALEZ, M. (2002), La teoría de las Relaciones Internacionales en los albores del siglo XXI: diálogo, disidencia, aproximaciones, Revista CIDOB d'Afers Internacionals, $\mathrm{n}^{\circ} 56, \quad$ p. 7-52. Disponible en línea: https://www.cidob.org/ca/articulos/revista_cidob_d_afers_internacionals/la_teoria_de_las _relaciones_internacionales_en_los_albores_del_siglo_xxi_dialogo_disidencia_aproxima ciones Consulta: 23 de Marzo de 2018

VIEIRA POSADA, E. (2005), Evolución de las Teorías sobre Integración en el Contexto de las Teorías de Relaciones Internacionales, Papel Político, $\mathrm{N}^{\circ} 18$, diciembre, $\mathrm{p}$. 235-290. Disponible en: https://www.redalyc.org/articulo.oa? $i d=77720389010$ Consulta: 23 de Marzo de 2018

VIVA, J. (2011), MERCOSUR Cultura. Revista Pampa, No7, p. 159-169. Disponible en: https://bibliotecavirtual.unl.edu.ar/publicaciones/index.php/PAMPA/article/view/3195 Consulta: 27 de Mayo de 2018

WORTMAN, Ana (2017). Democratización cultural en contextos de desigualdad social: políticas culturales como políticas sociales. Democracia e integración 25. Centro de Estudios Constitucionales

Sitios web consultados

IPPDH, Informe anual 2017, http://www.ippdh.mercosur.int/wpcontent/uploads/2017/12/Informe-IPPDH-2017Final.pdf

IPPDH Informe primer semestre 2018, http://www.ippdh.mercosur.int/wpcontent/uploads/2018/06/IPPDH-MERCOSUR-INFORME-PRIMER-SEMESTRE2018.pdf

MERCOSUR www.mercosur.int

MINISTERIO DE CULTURA DE LA REPÚBLICA ARGENTINA, www.cultura.gob.ar/institucional/organismos/ministerio-de-cultura/, www.cultura.gob.ar/institucional/organismos/secretaria-de-patrimonio-cultural/

MINISTERIO DE LAS CULTURAS, LAS ARTES Y EL PATRIMONIO DE CHILE, www.cultura.gob.cl 

Y CULTURALES
https://www.refworld.org.es/publisher,CESCR,GENERAL, 4ed35beb2,0.html

NACIONES UNIDAS: COMITÉ DE DERECHOS ECONÓMICOS, SOCIALES

(CESCR),

ORGANIZACIÓN DE LOS ESTADOS AMERICANOS, http://www.oas.org RAADH, COMISIÓN PERMANENTE EDUCACIÓN Y CULTURA EN DERECHOS HUMANOS, $\quad$ http://www.raadh.mercosur.int/comisiones/educacion-y-cultura-enderechos-humanos/?cp_educacion-cultura $=2$

SISTEMA ARGENTINO DE INFORMACIÓN JURÍDICA, www.saij.gob.ar 